\title{
FACTORES ASOCIADOS AL USO DE LA CITOLOGÍA CERVICOUTERINA EN ESTUDIANTES DE ENFERMERÍA DE LA CIUDAD DE CARTAGENA
}

Irma Yolanda Castillo Ávila ${ }^{1}$, Yelin Sepúlveda Mendoza² ${ }^{2}$ Laura Vergara Hernández ${ }^{2}$, Yuranis Zambrano Martínez ${ }^{2}$, María Camila Carval Salcedo ${ }^{2}$, Jessica Álvarez Puello ${ }^{2}$

\section{Resumen}

Introducción: El uso adecuado del tamizaje cérvico-uterino se configura como la mejor estrategia para la detección temprana del cáncer. Objetivo: determinar los factores asociados al uso de la citología cérvico-uterina en estudiantes de enfermería de la ciudad de Cartagena. Métodos: estudio analítico, con población de 1109 estudiantes y tamaño de muestra de 469, seleccionadas por muestreo polietápico. Se utilizó un modelo de regresión para determinar las variables explicativas de la utilización adecuada de la citología. Resultados: Participaron 469 estudiantes, promedio de edad 20.8 años $(\mathrm{DE}=3,4)$. El 50.7\% (238) ha realizado la prueba alguna vez, solo $31.3 \%$ (147) tienen uso adecuado. Ser mayor de 20 años (OR: 3,3 CI: 2,2 - 5,0), pertenecer a semestres superiores (OR: 1,9 CI: 1,3-2,9), tener pareja (OR: 3,1 CI: 1,9 - 5,0), tener hijos (OR: 2,9 CI: 1,8 - 4,7), haber estado embarazada (OR: 3,4 CI: 2,2 - 5,3), haber sufrido abortos (OR: 2,8 CI: $1,3-5,8)$ se asocian al uso adecuado. Conclusiones: Factores personales, principalmente antecedentes gineco-obstétricos, son predictores de la utilización adecuada de la citología.

Palabras clave: estudiantes de enfermería, cuello del útero, tamizaje, papanicolaou, utilización, neoplasias del cuello uterino. Fuente: (DeCS).

\footnotetext{
${ }^{1}$ Enfermera, Magíster en Salud Pública. Profesor Asociado, Facultad de Enfermería, Universidad de Cartagena, Colombia. icastilloa@unicartagena.edu.co

${ }^{2}$ Enfermera, Universidad de Cartagena, Colombia.
} 


\section{FACTORS ASSOCIATED WITH THE USE OF CERVICOUTERINE CYTOLOGYTEST IN NURSING STUDENTS OF THE CITY OF CARTAGENA}

Irma Yolanda Castillo Ávila ${ }^{1}$,Yelin Sepúlveda Mendoza², Laura Vergara Hernández ${ }^{2}$, Yuranis Zambrano Martínez ${ }^{2}$, María Camila Carval Salcedo ${ }^{2}$, Jessica Álvarez Puello

\section{Abstract}

Introduction: The appropriate use of Cervic-uterine screening test is configured as the best strategy for the early detection of cancer. Objetive: To determine the factors associated with the use of cervicaluterine cytology test in nursing students in the city of Cartagena. Methods: Analytical study, with a population of 1,109 subjects and sample size of 469 , selected by multi-stage sampling. A regression model to determine the explanatory variables of the adequate use of cytology test was applied. Results: 469 students on average age of 20.8 years $(S D=3.4)$ participated. $50.7 \%(238)$ have done the test at some time, only $31.3 \%$ (147) is used adequately. Being older than 20 years (OR: 3.3 CI: 2.2 - 5.0), belong to higher semesters (OR: 1.9 CI: 1.3 2.9), having a partner (OR: 3, 1 CI: 1.9 - 5.0), having children (OR: $2.9 \mathrm{CI}: 1.8$ - 4.7), having been pregnant (OR: $3.4 \mathrm{CI}: 2.2$ - 5.3), having practiced abortions (OR: $2.8 \mathrm{CI}: 1.3-5.8$ ) are associated with an adequate use. Conclusions: Personal factors, mainly gynecologicalobstetric history, are predictors of an adequate use of cytology test. 


\section{FATORES ASSOCIADOS AO USO DA CITOLOGIA CERVICAL EM ESTUDANTES DE ENFERMAGEM DA CIDADE DE CARTAGENA}

Irma Yolanda Castillo Ávila ${ }^{1}$,Yelin Sepúlveda Mendoza², Laura Vergara Hernández ${ }^{2}$, Yuranis Zambrano Martínez² , María Camila Carval Salcedo², Jessica Álvarez Puello

\section{Resumo}

Introdução: $\mathrm{O}$ uso adequado da triagem cervical configura-se como a melhor estratégia para a detecção precoce do câncer. Objetivo: : Determinar os fatores associados ao uso da citologia cervical em estudantes de enfermagem na cidade de Cartagena. Métodos: Estudo analítico, com uma população de 1.109 alunos e tamanho de amostra de 469, selecionados por amostragem em múltiplos estágios. Um modelo de regressão foi utilizado para determinar as variáveis explicativas do uso adequado da citologia. Resultados: Participaram 469 estudantes, com idade média de 20,8 anos ( $\mathrm{DP}=3,4) .50,7 \%(238)$ fizeram o teste em algum momento, apenas 31,3\% (147) tiveram uso adequado. Ser maior de 20 anos (OR: 3,3 IC: 2,2 - 5,0), pertencer a semestres superiores (OR: 1,9 IC: 1,3 - 2,9), ter companheiro (OR: 3, 1 IC: 1,9 - 5,0), ter filhos (OR: 2,9 IC: 1,8 - 4,7), ter estado gravida (OR: 3,4 IC: 2,2 - 5,3), ter sofrido abortamentos (OR: 2,8 IC: 1,3 5,8 ) estão associados ao uso adequado. Conclusões: Fatores pessoais, principalmente antecedentes gineco-obstétricos, são preditores do uso adequado da citologia. 


\section{Introducción}

La citología cérvico-uterina (CCU), también conocida como prueba de Papanicolaou (Pap), es un método para la detección precoz de lesiones intraepiteliales pre malignas progresivas del cérvix que tiene una alta especificidad, pero baja sensibilidad, y cuya realización periódica reduce de manera importante la morbilidad y mortalidad por esta neoplasia $(1,2)$.

Sin embargo, aunque este tamizaje se promueve desde hace muchos años, según datos de la Organización Mundial de la Salud (OMS), 230000 mujeres mueren anualmente por este tipo de cáncer; de estas, el $80 \%$ corresponde a mujeres de América Latina y África, y se prevé que este número de muertes se duplicará para el año $2030(3,4)$.

En Colombia, a partir de la expedición de la Resolución 00412 de 2000, se establece como norma técnica para la detección temprana del cáncer de cérvix la realización de la CCU a todas las mujeres entre 25 y 69 años, o aquellas menores de 25 años con vida sexual activa, siguiendo un esquema 1-1-3. Pese a esto, y a la facilidad del acceso a esta prueba, la proporción de cumplimiento de este esquema es muy bajo ubicando al carcinoma de cuello uterino como el segundo tipo de cáncer que más afecta a las mujeres del país (5). Según cifras del Instituto Nacional de Cancerología, el cáncer de cérvix presenta una incidencia de 36,8 casos por cada 100000 habitantes, hecho que constituye un problema

de salud pública $(6,7)$. En Cartagena la tasa de mortalidad por cáncer de cérvix para el año 2013 fue de 9,4 casos por cada 100000 (8-19).

Algunas de las principales razones por las que las mujeres no son regulares o no se someten a la prueba de tamizaje para el cáncer de cérvix, son el temor a ser diagnosticadas con cáncer, la vergüenza a ser examinadas, y/o la pereza o descuido personal $(11,12)$. En contraste, tener antecedentes familiares de cáncer de cérvix, haber padecido una Infección de Transmisión Sexual (ITS), encontrarse en un rango de edad alto y estar activa en el régimen de seguridad social son factores que contribuyen a la práctica regular de la prueba (13).

Así mismo, en la población de mujeres con mayores conocimientos sobre temas de salud, como por ejemplo las estudiantes de enfermería, se han descrito factores similares para la no realización de la prueba; siendo los principales argumentos, según lo reportado por algunos autores, el descuido $(14,15)$, el miedo a padecer cáncer, los sentimientos de pudor y la falta de tiempo relacionada con la carga académica excesiva del programa (16,17). En contraste, algunos aspectos que favorecen la toma de la prueba son: estar casada o tener pareja permanente, utilizar métodos de planificación familiar hormonal, tener hijos o haber estado embarazada por lo menos una vez (16).

Teniendo en cuenta lo anterior, y que pese a todas las políticas nacionales encaminadas al fortalecimiento de los programas de detección temprana del cáncer de cérvix (9), la utilización adecuada de esta prueba de tamizaje mues- 
tra resultados deficientes incluso entre las estudiantes de enfermería de quienes se esperaría, por el conocimiento científico adquirido durante los años de formación profesional, más regularidad en esta práctica (17). En este sentido, el estudio se propuso determinar los factores asociados al uso de la CCU en estudiantes de enfermería de la ciudad de Cartagena.

\section{Materiales y métodos}

Estudio analítico tipo encuesta transversal, con una población de 1109 estudiantes de enfermería, distribuidas en tres programas de la ciudad. El tamaño de muestra estimado fue de 482 , seleccionadas mediante un muestreo aleatorio en varias etapas, que incluyó estudiantes matriculadas de 25 a 69 años, o menores de 25 años con vida sexual activa. La recolección de la información se realizó mediante la aplicación de dos instrumentos: una encuesta sociodemográfica que valora variables como: edad, tipo de universidad a la que pertenece, semestre en curso, estado civil y antecedentes ginecobstétricos. Además, se utilizó el aparte para CCU de la Encuesta Nacional de Demografía y Salud (ENDS) 2010 que incluye preguntas como: ¿alguna vez se ha realizado una CCU?, ¿cuántas se han realizado?, ¿cuál fue la fecha de la última prueba?, ¿con qué frecuencia se la hace? y ¿reclamó o no el resultado?; así mismo, se incluyen preguntas para identificar las razones por las cuales las estudiantes se realizan o no la CCU.

La información recolectada fue incluida en una base de datos de Microsoft Excel y se procesó en el software SPSSS versión 22.0 para realizar el análisis bivariado y multivariado, previa dicotomización de las variables dependientes (utilización de la citología según el esquema propuesto en la norma técnica colombiana) e independientes (variables sociodemográficas y ginecobstetricias). Se calcularon OR e intervalos de confianza para determinar la asociación estadística entre las variables.

Esta investigación se realizó teniendo en cuenta los estatutos legales y normativos que rigen la investigación en Colombia. Se tuvo en cuenta la Declaración de Helsinki (18) que propone principios éticos y la Resolución No 008430 de 1993 (19), que instaura normas científicas, técnicas y administrativas para la investigación en salud; además, se diligenció el consentimiento informado por escrito. El estudio fue aprobado por el Comité de Investigación de la Facultad de Enfermería de la Universidad de Cartagena y avalado, para su aplicación, por los otros dos programas de enfermería de la ciudad.

\section{Resultados}

\section{Características sociodemográficas \\ de las estudiantes de enfermería participantes}

Un total de 469 estudiantes completaron satisfactoriamente la encuesta. El promedio de edad fue de 20,8 años $(\mathrm{DE}=3,4), 71.4 \%$ [335] pertenecían a los estratos 2 y 3, y la mayoría (51.7\% [243]) cursaban de $5^{\circ}$ a $9^{\circ}$ semestre. El 79.9\% [375] son solteras, $22.8 \%$ [107] se ha embarazado, $7.0 \%$ [33] ha 
INVESTIGACIONES ANDINA No. 35, Vol. 19

hijos. El 98.1\% [460] de la población se el régimen contributivo con un 56.9\% encuentran afiliadas al SGSSS, siendo [267] el de mayor proporción. (Tabla 1).

Tabla 1. Distribución según características sociodemográficas de las estudiantes participantes en el estudio. Cartagena.

\begin{tabular}{c|c|c}
\hline Indicadores & $\mathbf{n}$ & $\%$ \\
\hline Estrato & & \\
\hline 1 & 110 & 23,5 \\
2 & 200 & 42,6 \\
3 & 135 & 28,8 \\
4 & 21 & 4,5 \\
5 & 2 & 0,4 \\
6 & 1 & 0,2 \\
\hline
\end{tabular}

\begin{tabular}{ccc}
\hline Semestre & & \\
\hline I & 60 & 12,8 \\
II & 49 & 10,4 \\
III & 60 & 12,8 \\
IV & 57 & 12,2 \\
V & 61 & 13,0 \\
VI & 55 & 11,7 \\
VII & 62 & 13,2 \\
VIII & 56 & 11,9 \\
IX & 9 & 1,9 \\
\hline
\end{tabular}

\begin{tabular}{ccc}
\hline Estado Civil & \multicolumn{1}{c}{} \\
\hline Soltera & 375 & 79,9 \\
Casada & 44 & 9,4 \\
Separada & 1 & 0,2 \\
Divorciada & 3 & 0,6 \\
Unión libre & 46 & 9,8 \\
\hline Hijos & \\
\hline Si & \multicolumn{1}{c}{19,4} \\
No & 91 & 80,6 \\
\hline Embarazos & 378 & 22,8 \\
\hline Si & 107 & 77,2 \\
No & 362 & 7,0 \\
Abortos & 33 \\
Si No & 436 & 93
\end{tabular}


Continuación Tabla 1. Distribución según características sociodemográficas de las estudiantes participantes en el estudio. Cartagena.

\begin{tabular}{ccc}
\hline Indicadores & $\mathbf{n}$ & $\%$ \\
\hline Ocupación & & \\
\hline Estudiante & 413 & 88,1 \\
Estudiante y empleado & 56 & 11,9 \\
\hline Procedencia & & 33,5 \\
\hline Rural & 157 & 66,5 \\
Urbana & 312 & \\
\hline Afiliación en salud & & 32,0 \\
\hline Subsidiada & 150 & 56,9 \\
Contributiva & 267 & 1,9 \\
No tiene afiliación & 9 & 9,2 \\
Especial & 43 & $\mathbf{1 0 0}$ \\
\hline Total & $\mathbf{4 6 9}$ &
\end{tabular}

Fuente: Encuestas del Estudio.

\section{Uso de la CCU en estudiantes de enfermería encuestados}

El 50,7\% [238] de las participantes del estudio se han realizado la CCU, con una mediana de 2; pero solo 31,3\% [147] presentaron un uso adecuado (según el esquema 1-1-3), siendo mayor este porcentaje en los últimos semestres $(38.3 \%$ [93]) (Tabla 2).

Tabla 2. Distribución según realización de CCU y utilización adecuada de la misma en las estudiantes participantes en el estudio. Cartagena.

\begin{tabular}{|c|c|c|}
\hline Realización de CCU & $\mathbf{N}$ & $\%$ \\
\hline $\mathrm{Si}$ & 238 & 50,7 \\
\hline No & 231 & 49,3 \\
\hline Total & 469 & 100.0 \\
\hline Uso adecuado de la CCU & $\mathrm{N}$ & $\%$ \\
\hline Adecuado & 147 & 31,3 \\
\hline Inadecuado & 322 & 68,7 \\
\hline Total & 469 & 100.0 \\
\hline
\end{tabular}


El 39.9\% [95] de las encuestadas se realizaron la CCU a través de la EPS, 62.2\% [148] expresaron que esta última fue pagada totalmente por la institución. El 5\% [12] no reclamaron el resultado, siendo la principal razón temor a que le digan que tienen cáncer con un 33.3\% [4]. El 59.2\% [141] se realizó la prueba por intención propia, $8.8 \%$ [21] por seguimiento del esquema, 7.1\% [17] por estar embarazada y un 3.8\% [9] por infecciones vaginales previas. El 29,8\% [71] no se han realizado una nueva CCU porque creen que pueden esperar, 16.4\% [39] no saben por qué, $13,9 \%$ [33] manifestaron que los horarios de consulta no le sirven y $11.8 \%$ [28] por pereza o descuido. Una gran proporción de las estudiantes expresaron que nunca se han realizado la CCU por falta de tiempo 29.8\% [69], seguido de pereza /descuido con un $22.1 \%$ [51] (Tabla 3).

\section{Relación entre el uso adecuado de la CCU y algunos factores presenten en la población}

En relación a la edad, se encontró que superar los 20 años se asocia con una mayor probabilidad del uso adecuado de la CCU. Esta relación es significativa (OR: 3,3 CI: 2,2 - 5,0). Los estudiantes de los últimos semestres de la carrera tienen mayor oportunidad de usar adecuadamente la prueba de tamizaje $(38,3 \%)$, que aquellos que se encuentran en los primeros semestres $(23,9 \%)$. Esta relación es significativa (OR: 1,9 CI: 1,3 $-2,9)$.

Quienes tienen pareja mostraron mayor proporción de uso adecuado de la CCU $(52,7 \%)$ que aquellas sin pareja $(26,2$ $\%)$. Esta relación es significativa (OR: 3,1 CI: 1,9-5,0). Del mismo modo, las estudiantes con hijos (OR 2,9 IC (1,8 $4,7)$ ), que han estado embarazadas (OR $3,4$ IC $(2,2-5,3))$ y quienes han sufrido un aborto (OR 2,8 IC $(1,3-5,8)$ ) tienen mayores probabilidades de presentar utilización adecuada de la prueba.

\section{Factores explicativos del uso de la citología de acuerdo al esquema propuesto por la norma técnica}

Las variables que resultaron significativas en el análisis bi-variado se sometieron a un modelo de regresión, del cual, ser mayor de 20 años y haber estado en embarazo fueron las variables que explicaron el uso adecuado de la CCU en las estudiantes de enfermería de la ciudad de Cartagena. (Tabla 3).

Tabla 3. Factores explicativos del uso de la citología según el esquema propuesto por la norma técnica en las estudiantes de enfermería. Cartagena 2013.

\begin{tabular}{lccc}
\hline & & \multicolumn{2}{c}{ I.C. 95\% } \\
\cline { 3 - 4 } Variables & OR & Límite Inferior & Límite Superior \\
Mayor de 20 años & 2,393 & 1,518 & 3,774 \\
Ha estado embarazada & 5,219 & 1,101 & 24,731 \\
\hline
\end{tabular}




\section{Discusión}

Los factores que explican el uso adecuado de la CCU, en las estudiantes de enfermería de Cartagena, son la edad mayor de 20 años y haber estado en embarazo.

Como se muestra en este estudio, la proporción de la toma de la prueba entre las estudiantes encuestadas, 50,7\% [238] es superior a la encontrada en la Universidad de Carabobo en Venezuela donde solo el 32\% de las estudiantes han accedido al tamizaje cérvico-uterino (17). Sin embargo, es ligeramente inferior a la reportada por Guevara C (16) en estudiantes del área de la salud de una universidad pública de Cali, donde el porcentaje de toma de CCU, alcanza el 56\%. Se esperaría que un número mayor de estudiantes universitarias se realizara la CCU, para corroborar lo planteado por Cogollo Z, quien a partir de los datos obtenidos en su estudio, refuerza que un mayor porcentaje de prácticas de cuidado de la salud relacionadas con el acceso regular al tamizaje para cáncer de cuello uterino, estaría influenciado por un mayor grado de conocimientos sobre este y mediado por actitudes favorables de las mujeres frente a la prueba (1).

La mitad de la población participante admitió haberse realizado la CCU, sin embargo, solo la tercera parte de ellas mantuvieron un correcto uso de esta práctica. Se resalta que estas estudiantes están matriculadas, en su mayoría, desde 5to semestre en adelante siendo este factor predictor del uso adecuado de la misma. Estos datos sugieren que la formación en salud no cambia de forma relevante algunas actitudes y prácticas culturales socialmente aceptadas y consolidadas.

Además, es importante considerar que un grupo sustancial de estudiantes ingresa a la carrera en la adolescencia media o tardía, sin una amplia consolidación de patrones de comportamientos relacionados con la salud general y la salud sexual-reproductiva; lo que representa una disyuntiva, que si bien implica muchas conductas de riesgo, puede constituirse en una oportunidad para abrir paso a la formulación de políticas para la promoción de la salud en el contexto universitario, que ayuden a fomentar el ejercicio de la sexualidad de manera responsable, el auto-cuidado y la conservación del bienestar sexual. Todas estas acciones demandan la conjugación de esfuerzos desde los diferentes actores institucionales: políticas de salud, planes estratégicos directivos, rol de los docentes en la promoción de la salud y la integración de estos contenidos a la estructura curricular (20-23).

Ser menor de 20 años reduce de manera significativa el acceso al tamizaje regular mediante la práctica de la citología. Según la ENDS del año 2015 (12), la práctica de la citología se incrementa con la edad, el nivel educativo y el índice de riqueza. Esta situación muestra la necesidad de formación y fortalecimiento de los programas de demanda inducida desde los centros de salud del primer nivel de atención. La misma encuesta evidencia que para los jóvenes del país, la vida sexual inicia a edades más tempranas y que pese a ello, el acceso a la información o estrategias relacionadas 
con la prevención de infecciones es muy reducido. Lo anterior, debido a que en ese rango de edad no existe percepción de riesgo de afecciones cérvico-uterinas, tal y como lo reporta Da Cruz D $(14,15,23)$, en un estudio realizado con adolescentes en Brasil, donde la mayoría de las adolescentes que había iniciado su vida sexual no accedía a la prueba por descuido, por no considerarlo necesario, o por vergüenza.

También se desatacan como factores explicativos del uso adecuado de la citología, haber estado en embarazo, tener hijos, haber sufrido abortos y tener pareja; dato que puede relacionarse con la planteado por Guevara C, en Cali quien encontró asociación estadísticamente significativa entre el número de relaciones sexuales por mes y la práctica de CCU. Esta asociación podría explicarse porque las mujeres de más edad relacionan el incremento de la actividad sexual coital, con más riesgo para contraer infecciones de transmisión sexual y con tener alteraciones cervicales. Además, la norma técnica nacional establece que debe realizarse prueba de tamizaje cérvico-uterino para las mujeres embarazadas, si no tienen ninguna condición de riesgo, lo que explica una mayor proporción de realización de la prueba entre las estudiantes que han estado en embarazo; incluso si este no llegó a feliz término $(5,16,24,25)$.

La periodicidad en la realización de la práctica fue, en su mayoría, anual $(14,16,17,23)$. Si bien es positivo encontrar que, un alto porcentaje de mujeres 54 accede al tamizaje con la frecuencia recomendada por las diferentes asocia- ciones nacionales e internacionales para la prevención del cáncer de cérvix, las cifras reportadas en la reclamación de resultados son bajas. Un número importante de encuestadas muestran baja proporción de reclamación de los resultados lo cual, según lo expresado por ellas, se debe a falta de tiempo por la sobrecarga académica y a las limitaciones institucionales de los horarios $(16,24,25)$.

La mayor proporción de las estudiantes expresaron nunca haberse realizado la prueba CCU por falta de tiempo, seguido de pereza/descuido, miedo/temor y la percepción de poca calidad de la atención en el servicio; dato consistente con lo reportado por múltiples investigadores. Por ejemplo, Espinosa L. (24), en una investigación con universitarias de Santander, identificó como factores relacionados a la no utilización de servicio de CCU, la pena o vergüenza, seguida de falta de tiempo, temor, dolor e incomodidad y a no tener vida sexual activa. Así mismo, lo reportado por Comunián G. en estudiantes de educación superior en Venezuela, describe dentro de las principales razones para no hacerse la CCU el miedo, la falta de tiempo, la falta información sobre la prueba, la vergüenza, las limitaciones económicas para cubrir el costo de la misma, y vivir lejos del consultorio (17). Por su parte, Ybarra J, en un estudio realizado con universitarias en Méjico, informó que los principales motivos para no acceder al tamizaje fueron la vergüenza, la falta de tiempo y la falta de información (25). $\mathrm{Al}$ ser comparados estos datos con la población de mujeres colombianas, en general siguen prevaleciendo las mismas razones para no acceder a esta práctica. 
Según el reporte de la ENDS 2015(12) las principales razones expuestas para no hacerse el examen son no considerarlo importante o necesario, el miedo y el descuido.

Si bien las estudiantes se han realizado la CCU de forma regular por intención propia, por seguimiento del esquema, por encontrarse en embarazo o por infecciones vaginales previas; los datos reportados por el estudio difieren del realizado por Ybarra J, donde las universitarias solían acceder a la práctica en una mayor proporción, cuando esta era ordenada por el médico; seguida de razones como la prevención de ETS y el consejo de la pareja. Estas diferencias resaltan aspectos importantes como el apoyo familiar y de la pareja como una influencia positiva para el cuidado de la salud de la mujer, y la relación entre las pacientes y los servicios de salud, a partir de la promoción del acercamiento entre los usuarios a los programas de tamizaje (25).

En general, estos resultados permiten tener un panorama amplio de las prácticas relacionadas con el autocuidado en las estudiantes de las distintas Facultades de Enfermería de la ciudad de Cartagena, en relación con el tamizaje para cáncer de cuello uterino. De lo anterior, se destaca la necesidad de fortalecer los programas de promoción y prevención desde el contexto universitario con el propósito de permitir la exploración de aquellas barreras que limitan el aumento de cobertura en la toma de la CCU, puesto que son estas estudiantes quienes deben ser sujetos ejemplarizantes en la educación e instrucción sobre la impor- tancia de adquirir hábitos de vida saludables, de acuerdo con su función profesional.

Las principales limitaciones del estudio se relacionan con las características del diseño y con aquellas derivadas del auto-reporte.

\section{Conclusiones}

Se concluye que las estudiantes de enfermería objeto del estudio no hacen un uso adecuado de esta práctica, debido a que no se realizan la prueba de acuerdo con lo establecido en la norma técnica para la detección temprana del cáncer del cuello uterino del Ministerio de Salud. Según el estudio, los factores principales por los que las estudiantes se realizaron la CCU son intención propia, seguimiento del esquema, recomendación del médico, estado de embarazo y por haber contraído infecciones vaginales previas. Fueron más las razones encontradas para el no uso de la CCU, dentro de las cuales sobresalen la falta de tiempo, la pereza/descuido, el miedo o temor a ser diagnosticadas, razones que involucran la calidad de la atención del servicio y la tendencia a postergar que se encuentra relacionada con el seguimiento del esquema. Por otro lado, aspectos personales como una mayor edad y antecedentes de embarazo, son los predictores de la utilización adecuada de la prueba.

Este estudio permite mayores insumos para comprender los aspectos que facilitan o limitan el acceso al tamizaje para cáncer de cérvix entre la población de enfermeras en formación de la ciudad de Cartagena. 


\section{Referencias}

1. Cogollo Z., Castillo I., Torres B., et al. Conocimientos, actitudes y prácticas de mujeres entre 18 y 49 años frente a la citología cérvico-uterina en instituciones de salud pública de Cartagena (Colombia). Salud Uninorte. Barranquilla (Col.) 2010; 26 (2): 223-231.

2. Organización Mundial de la Salud. Intervenciones dirigidas a mujeres para alentar la captación en el tamizaje cervical [Internet]. Disponible en: http://apps.who. int/rhl/gynaecology/cancer/mjgguide/es/ (Consultado 09 febrero de 2017).

3. Organización Panamericana de la Salud. Análisis de la situación del Cáncer Cervico-uterino en América Latina y el Caribe [Internet]. [Citado el 15 noviembre 2017].

4. Disponible en: http://www1.paho.org/ spanish/ad/dpc/nc/pcc-cc-sit-lac.pdf

5. Pan American health organization regional office of the world health organization. 110 años 1902-2012. Cáncer cervicouterino en las Américas [Internet]. [Consultado 05 septiembre 2017]. Disponible en: (http://www.paho.org/hq/index. php?option $=$ com_docman\&task $=$ doc_ view\&gid=19818\& Itemid=).

6. Colombia. Ministerio de Protección social. Resolución número 00412 de 2000. Norma técnica para la detección temprana del cáncer de cuello uterino y guía de atención de lesiones pre-neoplásicas de cuello uterino [Internet]. [Consultado 15 noviembre 2017]. Disponible en: http:// server.esenorte.gov.co:82/Pagelntranet/ wp-content/uploads/2014/08/PP.E.08Deteccion-temprana-del-cancer-decuello-uterino.pdf

7. Combita A., Molano M., Muñoz M., Bravo $M$. Respuesta inmune humoral hacia los papiloma virus oncogénicos tipos 16 , 31 y 58 en mujeres colombianas con citología normal. Revista Colombiana de Cancerología, 2009; 13(2): 77-87
8. Colombia. Ministerio de Protección social. Indicadores básicos de salud 2012 [Internet]. [Consultado 2 febrero 2017]. Disponible en: https://www.minsalud.gov. $\mathrm{co} / \mathrm{sites} / \mathrm{rid} /$ Lists/BibliotecaDigital/RIDE/ VS/ED/PSP/Indicadores-basicos-en-salud-2012.pdf.

9. Departamento administrativo distrital de salud. DADIS Cartagena. Análisis de la mortalidad por neoplasias en el distrito de Cartagena año 2013 [Internet]. [Consultado 1 febrero de 2017]. Disponible en: http://www.dadiscartagena.gov.co/ images/docs/saludpublica/vigilancia/informe_de_mortalidad_neoplasias_2013.pdf

10. Ministerio de Salud. Plan Decenal Para el Control del Cáncer en Colombia. 2012-2021. [Consultado 20 febrero 2017]. Disponible en: https://www.minsalud.gov. co/DocumentosyPublicaciones/PlanDecenalparaelControldelCancer.pdf).

11. Alcaldía Mayor de Cartagena de Indias. Departamento Administrativo Distrital de Salud. Perfil Epidemiológico del distrito de Cartagena. Año 2014. [Consultado: 17 noviembre 2016, Citado 02 diciembre de 2016]. http://www.dadiscartagena. gov.co/images/docs/saludpublica/perfil_ epidemiologico_2014.pdf

12. Instituto nacional de cancerología. Plan nacional para el control del cáncer en Colombia 2010-2019 [Internet]. [Consultado 2 febrero 2017]. Disponible en: https:// www.minsalud.gov.co/Documentos $\% 20$ $\mathrm{y} \% 20$ Publicaciones/Plan $\% 20$ nacional $\% 20$ para $\% 20$ el $\% 20$ control $\% 20$ del $\% 20$ c $\%$ C3\%A1ncer\%20en\%20Colombia.pdf

13. Profamilia. Encuesta nacional de demografía y salud 2015 [Internet]. [Consultado 20 Noviembre 2017]. Disponible en: http://profamilia.org.co/docs/ENDS $\% 20$ \%20TOMO\%20I.pdf

14. Fajardo A., Méndez F., Molina L. Factores que inciden en la no realización de 
la citología vaginal en las mujeres Bogotanas. 2008. Investigaciones andina. 2008; 12 (21):61-69.

15. Bárcenas M., Delgado L., Gallegos R., et al. La perspectiva de estudiantes de enfermería para la realización del Papanicolaou. Lux Médica. 2014;(27): 41-7

16. Malak A., Yilmaz D., Tuna A., et al. Relations between breast and cervical cancer prevention behavior of female students at a school of health and their healthy life style. 2010; 11(1):53-6.

17. Guevara C., Guevara C., Medina C., et al. Prevalencia de la toma de citología vaginal en estudiantes de una universidad pública de Colombia. Salud Uninorte. Barranquilla (Col.) 2008; 24,(1):23-30.

18. Comunián G., Blanchard B., Briceño J., Martí A. Inasistencia a la consulta de despistaje de cáncer de cuello uterino en estudiantes universitarias. GacMéd Caracas 2008;116 (2):115-20.

19. Mazzanti M. Declaración de Helsinki, principios y valores bioéticos en juego en la investigación médica con seres humanos. Revista Colombiana de Bioética, 2011; 6 (1):125-44.

20. Colombia, Ministerio de Salud. Resolución 008430 de 1993. Por la cual se establecen las normas científicas, técnicas y administrativas para la investigación en salud. Bogotá: El Ministerio, 1993.

21. American Cancer Society. Cáncer de cuello uterino: Detección temprana y prevención [Internet]. [Consultado 16 noviembre de 2016]. Disponible en: http:// www.cancer.org/acs/groups/cid/documents/webcontent/002580-pdf.pdf).

22. Colombia. Congreso de la república de Colombia. Ley 1438, 19 de enero 2011 por medio de la cual se reforma el sistema general de seguridad social en salud y se dictan otras disposiciones. Bogotá: Congreso de la república de Colombia; 2011.

23. Campo A. Salud sexual y reproductiva en estudiantes de medicina en Colombia. Revista de la Facultad de Ciencias de la Salud Duazary. 2011; 8(2): 121-23

24. Da Cruz DE, Jardim DP. Adolescencia y Papanicolau: Conocimiento y Práctica. Adolesc Saude. 2013;10 (supl 1):34-42.

25. Espinosa L., Valdivieso J., Joya M. Factores influyentes en la utilización del servicio de citología en una universidad en Santander (Bucaramanga). Rev Cuid 2010;(1):19-25.

26. Ybarra J, Perez B, Romero D. Conocimiento y creencias sobre la prueba de $\mathrm{Pa}-$ panicolaou en estudiantes universitarios. Rev Psico y Salud 2012; 22 (2): 185-94. 
INVESTIGACIONES ANDINA No. 35, Vol. 19 